\title{
A case of perforated gallbladder with liver abscess
}

\author{
J.C.Y. Gan, K.K. Chan
}

Department of General Surgery, Hospital Sultanah Aminah, Johor Bahru, Johor, Malaysia.

Keywords: Perforated gall bladder; liver abscess

\section{Introduction}

Perforation of the gallbladder resulting in an intrahepatic abscess is rare. This condition is commoner when the gallbladder is completely or partially intrahepatic [1]. It is associated with a high morbidity and mortality and requires accurate diagnosis and aggressive treatment [2].

\section{Case report}

A 54 year old Chinese man with diabetes and hypertension and who had a recent attack of acute cholecystitis two weeks ago presented with intermittent upper abdominal pain associated with fever, chills and rigors for one week. During his prior hospitalization, he was treated with intravenous antibiotics and was discharged after four days with resolving symptoms. On admission, he appeared septic with a temperature of $38.5^{\circ} \mathrm{C}$, blood pressure of $120 / 70 \mathrm{mmHg}$, a pulse rate of 140 beats per minute and a capillary blood sugar level of $13.1 \mathrm{mmol} / \mathrm{L}$. On examination he was not icteric. The abdomen was tender with localized guarding at the right hypochondrium. There were no palpable masses at the right hypochondrium. His blood investigation revealed a normal total white cell count and a serum amylase level but the serum bilirubin (22umol/L) and alkaline phosphotase (212 U/L) were mildly elevated. Subsequently he had an urgent abdominal ultrasound scan performed which showed a hypoechoic lseion in segment $\mathrm{V}$ of the liver measuring $4.0 \times 11.3 \times 6.4 \mathrm{~cm}$ suggestive of an abscess with a calculus within and ruptured gall bladder empyema. He was resuscitated with intravenous fluid, kept nil by mouth, and intravenous co-amoxyclav $1.2 \mathrm{~g} 8$ hourly commenced. The abscess was further assessed with CECT abdomen prior to percutaneous drainage which showed the

Correspondence: J.C.Y. Gan

E-mail: cyjoan2020@gmail.com collection extending into segment $\mathrm{V}$ (Figure 1). Percutaneous drainage was done under ultrasound guidance within 24 hours of admission and it drained about $100 \mathrm{cc}$ of pus. The catheter was placed within the gall bladder. His blood culture grew Kleibsiella pneumoniae which was sensitive to the antibiotic used.

His condition improved significantly following the drainage. An ultrasound assessment was done on day 4 of admission which revealed a distended gallbladder with multiple calculi within but the liver abscess had reduced in size. He then underwent an open cholecystectomy.

Intraoperatively, the gall bladder was densely adhered to the surrounding structures especially at the abscess cavity and thus partial cholecystectomy was performed. Pus from the abscess cavity when cultured yielded growth of Kleibsiella pneumoniae and Aeromonas hydrophila which was sensitive to imipenem. The patient was discharged five days later following the surgery.

\section{Discussion}

This patient had type II gall bladder perforation which was described according to classification by Niemeier in $1934[3,4]$. The diagnosis is confirmed by

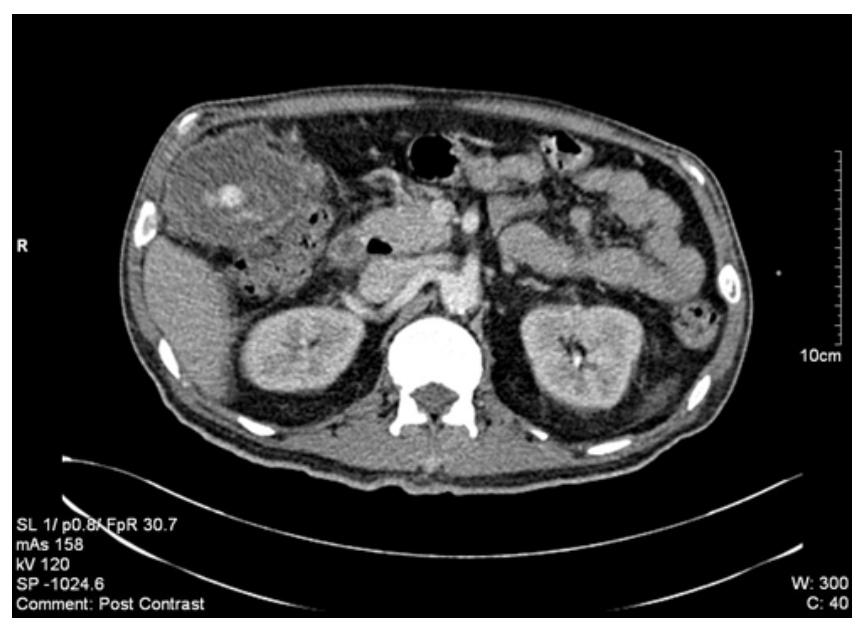

Figure 1. Gall bladder empyema with multiple gall stones 
radiological imaging e.g. ultrasonography or computed tomography with constrast study (CECT). Upon making the diagnosis, the decision for treatment is based mostly on the clinical features of the patient including patient's fitness for surgery at the time of diagnosis and the severity of the disease. Our patient underwent percutaneous gallbladder drainage (PTGBD) as it has been proven to be effective and relatively safe with lower risk of morbidity and mortality compared to emergency cholecystectomy [5]. By performing a percutaneous drainage prior to definitive surgery in fact provides the opportunity for optimization of a patient's condition including the comorbidity and the possibility of cholecystectomy via laparoscopic approach.

\section{References}

1. Peer A1, Witz E, Manor H, Strauss S. Intrahepatic abscess due to gallbladder perforation. Abdom Imaging. 1995 Sep-
Oct;20(5):452-5.

2. Derici H, Kara C, Bozdag AD. Diagnosis and treatment of gallbladder perforation. World J Gastroenterol. 2006;12(48):7832-36.

3. Anderson BB, Nazem A. Perforations of the gallbladder and cholecystobiliary fistulae: a review of management and a new classification. J Natl Med Assoc 1987; 79:393-399.

4. Ibrarullah M, Saxena R, Sikora SS, Kapoor VK, Kaushik SP. Unusual gallbladder perforation- - definition of a new type. Indian J Gastroenterol 1992; 11:170.

5. C-C Huang, H-C lo, Y-M Tzeng et al. Percutaneous transhepatic gall bladder drainage: a better initial therapeutic choice for patients with gall bladder perforation in the emergency department. Emerg Med J. 2007 December; 24(12):836-840.

\section{Key points:}

- Perforation of the gallbladder causing an intrahepatic abcess is rare.

- Performing a percutaneous drainage helps to optimise a patient's condition prior to definitive surgery. 